
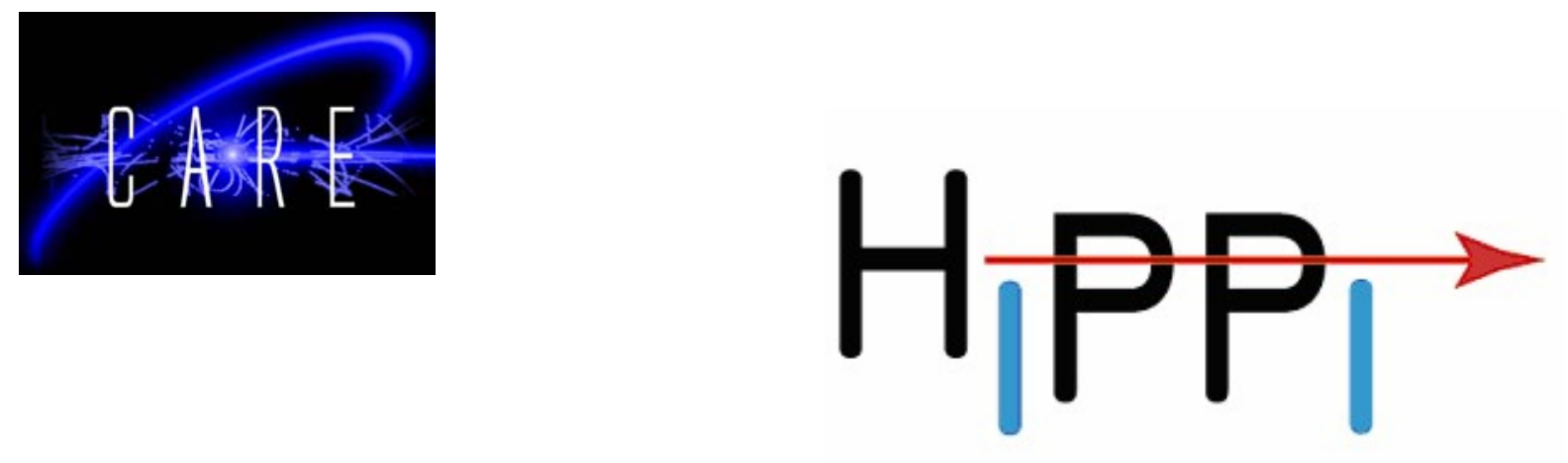

\title{
'FAST-SLOW' BEAM CHOPPING FOR NEXT GENERATION HIGH POWER PROTON DRIVERS
}

Michael A. Clarke-Gayther, CCLRC RAL, Didcot, United Kingdom

\begin{abstract}
A description is given of two 'state of the art' high voltage pulse generator systems, designed to address the requirements of a fast beam chopping scheme for next generation high power proton drivers [1]. Measurements of output waveform and timing stability, for fast transition short duration, and slower transition long duration pulse generators, are presented.
\end{abstract}

Contribution to the PAC05, Knoxville, USA

Work supported by the European Community-Research Infrastructure Activity under the FP6 "Structuring the European Research Area" programme (CARE, contract number RII3-CT2003-506395). 


\title{
'FAST-SLOW' BEAM CHOPPING FOR NEXT GENERATION HIGH POWER PROTON DRIVERS
}

\author{
Michael A. Clarke-Gayther, CCLRC RAL, Didcot, United Kingdom †.
}

\begin{abstract}
A description is given of two 'state of the art' high voltage pulse generator systems, designed to address the requirements of a fast beam chopping scheme for next generation high power proton drivers [1]. Measurements of output waveform and timing stability, for fast transition short duration, and slower transition long duration pulse generators, are presented.
\end{abstract}

\section{INTRODUCTION}

The development of an efficient beam chopper design is regarded as key for all next generation high intensity proton driver schemes that adopt the linac - accumulator or linac - synchrotron schemes [2]. Beam loss at ring injection and extraction, and the consequent activation of components can be minimised by the programmed population of ring longitudinal phase space, generated by a fast beam chopper in the linac front end. Chopper designs for the European Spallation Source (ESS), Spallation Neutron Source (SNS), Japan Proton Accelerator Research Complex (JPARC), and Superconducting Proton Linac (SPL), are the subject of a recent review paper [3].

ESS front-end specifications call for significant technical development as the design includes all of the key front-end elements (ion source, chopper, and funnel) required for next generation high intensity spallation and neutrino factories [4].

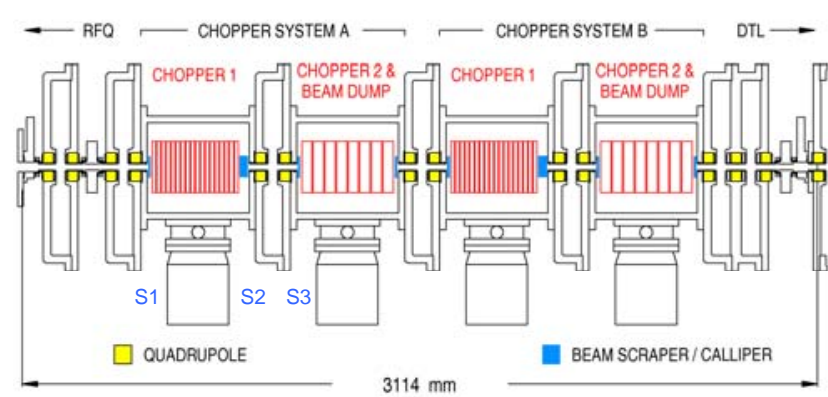

Figure 1: ESS 2.5 MeV MEBT with 'Tandem' chopper

A schematic drawing of an ESS MEBT line is shown in Figure 1. The configuration has evolved from a previously reported design [5], and utilises two slowwave E-field chopper systems operating in 'Tandem'. The scheme reduces beam dump power dissipation, and high voltage pulse generator repetition frequency by a factor of two, without incurring excessive emittance growth.

$\dagger$ Work supported by CCLRC/RAL/ASTeC and by the European Community-Research Infrastructure Activity under the FP6 "Structuring the European Research Area" programme (CARE, contract number RII3-CT-2003506395).

\section{'FAST-SLOW' CHOPPING SCHEME}

Key parameters and a timing schematic for one subsystem of the ESS 'Tandem' chopper configuration are shown in Table 1, and Figure 2, respectively.

Table 1: Key parameters for the ESS chopper system

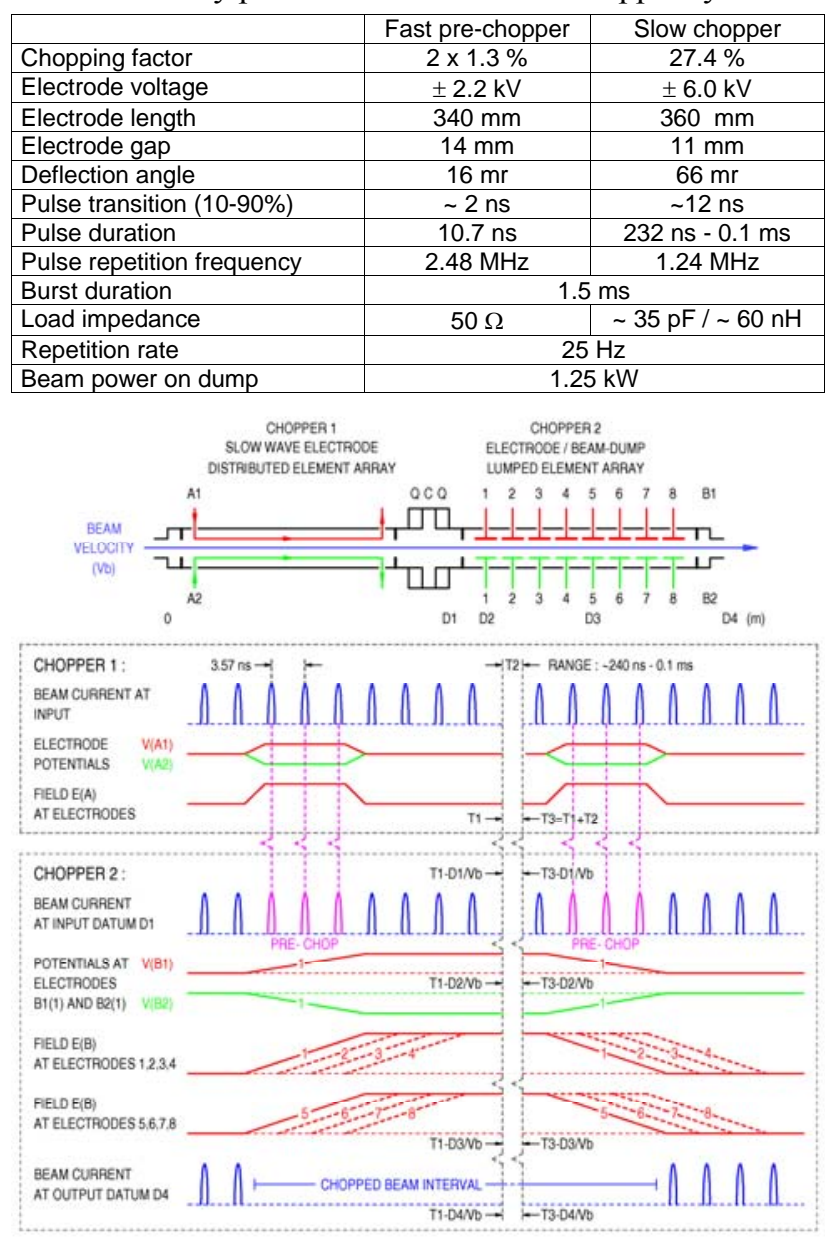

Figure 2: 'Fast-slow' chopper timing schematic

'Tandem' sub-systems are identical in operation and function alternately at a repetition frequency of $25 \mathrm{~Hz}$. Each sub-system consists of an upstream fast chopper with a slow-wave electrode structure [6], and a downstream (slower) main chopper with water-cooled lumped element electrodes, that also serve as a beam dump. Slow-wave chopper 1 produces a unipolar pulsed field that deflects just three adjacent bunches through $\sim 16 \mathrm{mr}$. into scrapers S2, S3 and chopper 2 beam dump electrodes, creating two $\sim 14$ ns duration gaps in the bunch train at the beginning and end of each chopped beam interval. These gaps ensure that no partially chopped bunches result from the slower field transition time of chopper 2 . 


\section{FAST \& SLOW PULSE GENERATORS}

A block schematic of the prototype high voltage pulse generator configuration for one chopper sub-system is shown in Figure 3.

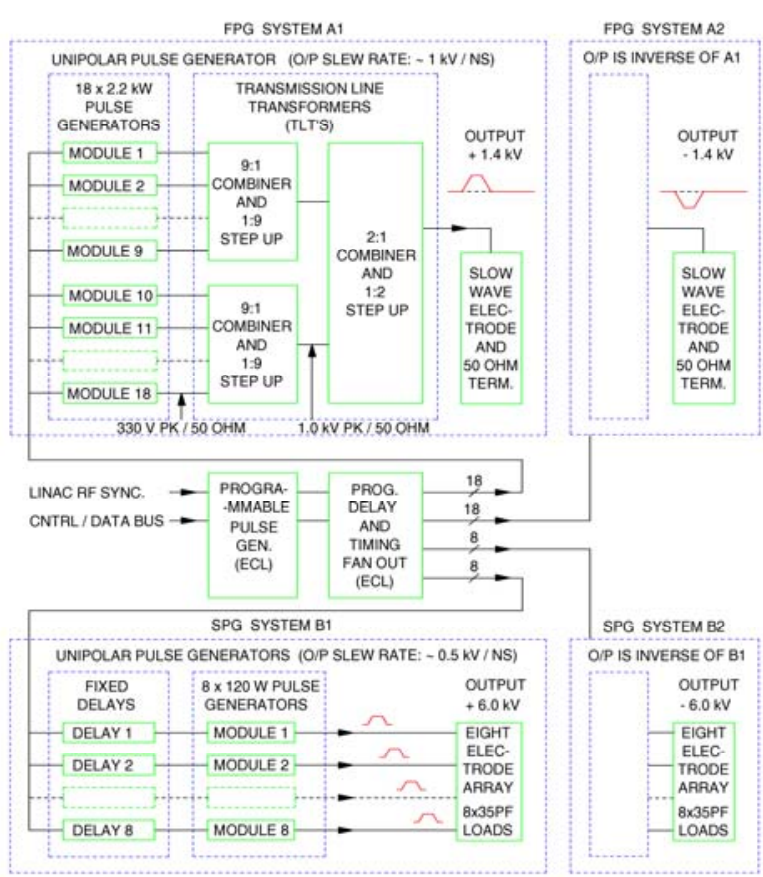

Figure 3: Fast and slow pulse generator block diagram

'Fast' pulse generator (FPG) systems A1 / A2 drive the A1 / A2 distributed slow-wave electrodes of chopper 1, as shown in Figures $2 \& 3$, and output fast transition ( 2 ns), short duration $(\sim 12 \mathrm{~ns})$ quasi-trapezoidal, unipolar high voltage pulses $( \pm 1.4 \mathrm{kV})$ into $50 \Omega$ loads. The modular configuration makes extensive use of high power transmission line transformers (TLT's) for efficient wide-band impedance transformation and combination of the outputs of 18 solid-state high voltage pulse generator cards, consisting of two, nine card modules. Additional modules and TLT's can be added, to increase output pulse amplitude to $\sim \pm 2.2 \mathrm{kV}$.

'Slow' pulse generator (SPG) systems B1 / B2 drive the B1 / B2 lumped element slow wave electrodes of chopper 2, and output $\sim 12$ ns transition, unipolar, trapezoidal, high voltage pulses $(+6.0$ and $-6.0 \mathrm{kV})$ into eight pairs of $35 \mathrm{pf} / 60 \mathrm{nH}$ loads. Pulse duration will be programmable in the $240 \mathrm{~ns}$ to $0.1 \mathrm{~ms}$ range. The $120 \mathrm{~W}$ fan-cooled modules will be close-coupled to the electrodes, to preserve pulse shape fidelity.

\section{FPG Development and waveforms}

The FPG has been designed and built by a UK based manufacturer [7]. Acceptance tests on the prototype indicated that with the exception of pulse droop, all key ESS specifications had been met [1]. TLT cores have now been replaced with an upgraded ferrite material and the pulse 'droop' specification ( $\leq 2 \%$ in $10 \mathrm{~ns})$ has subsequently been met. The system is now available for testing differential slow-wave structures, with dual polarity pulses of up to $1.4 \mathrm{kV}$ in amplitude.

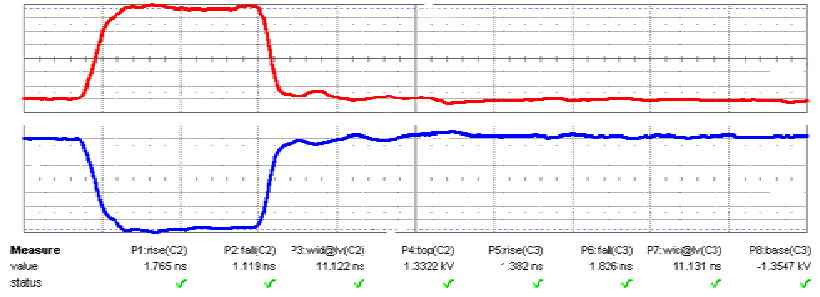

Figure 4: FPG waveforms at $\pm 1.4 \mathrm{kV}$ peak \& $5 \mathrm{~ns} / \mathrm{div}$.

The waveforms shown in Figure 4 indicate that the specification for transition time $(\leq 2 \mathrm{~ns})$ has been met. A reduction in post pulse aberration is anticipated.

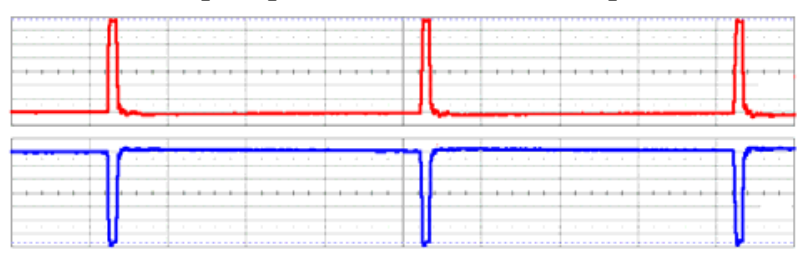

Figure 5: FPG waveforms at $\pm 1.4 \mathrm{kV}$ peak $\& 100 \mathrm{~ns} / \mathrm{div}$.

The waveforms shown in Figure 5 indicate that the requirements for pulse 'droop' $(\leq 2 \%$ in $10 \mathrm{~ns})$ and pulse repetition frequency $(\mathrm{PRF})(\geq 2.5 \mathrm{MHz})$ have been met.

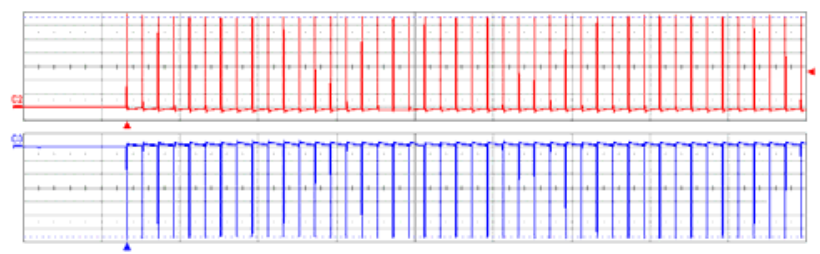

Figure 6: FPG waveforms at $\pm 1.4 \mathrm{kV}$ peak $\& 2 \mu \mathrm{s} /$ div.

The waveforms in Figure 6 show baselines settling in $\sim 5 \tau(\sim 2.5 \mathrm{e}-6 \mathrm{~s})$, confirming an $\mathrm{F}_{3 \mathrm{dblow}}$ of $\sim 300 \mathrm{kHz}$. A reduction in duty cycle 'droop' $(2.5 \%)$ is anticipated.

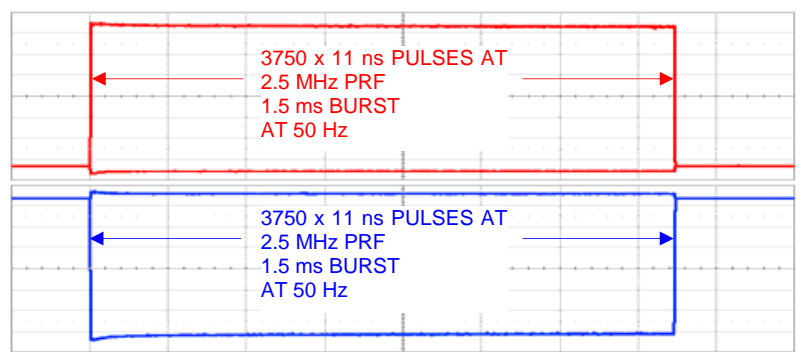

Figure 7: FPG waveforms at $\pm 1.4 \mathrm{kV}$ peak $\& 0.2 \mathrm{~ms} / \mathrm{div}$.

The waveforms shown in Figure 7 indicate that the requirements for duty cycle $(0.24 \%)$ and burst pulse amplitude stability $( \pm 5 \%$ ) have been met.

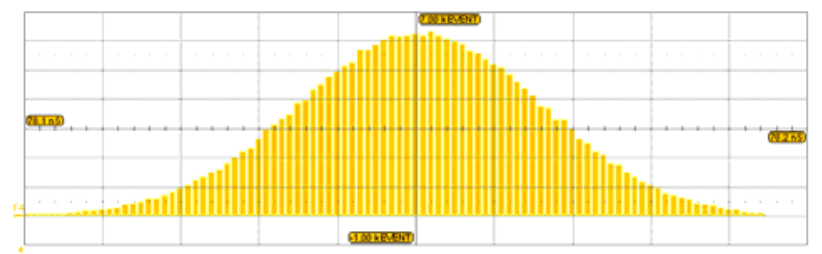

Figure 8: FPG timing 'jitter' at $10 \mathrm{ps} / \mathrm{div}$.

The histogram shown in Figure 8 indicates that the requirement for stability in delay $( \pm 0.1 \mathrm{~ns}$ pk-pk) between the trigger and output pulse has been met. 


\section{SPG Development \& waveforms}

The proposed 'slow' chopper structure will consist of eight electrode pairs, close-coupled to a 'phased array' of eight SPG pairs, as shown in Figures $2 \& 3$. Prototype testing is planned on a representative configuration of two adjacent SPG pairs, coupled to a 'dummy load' as shown in Figure 9.

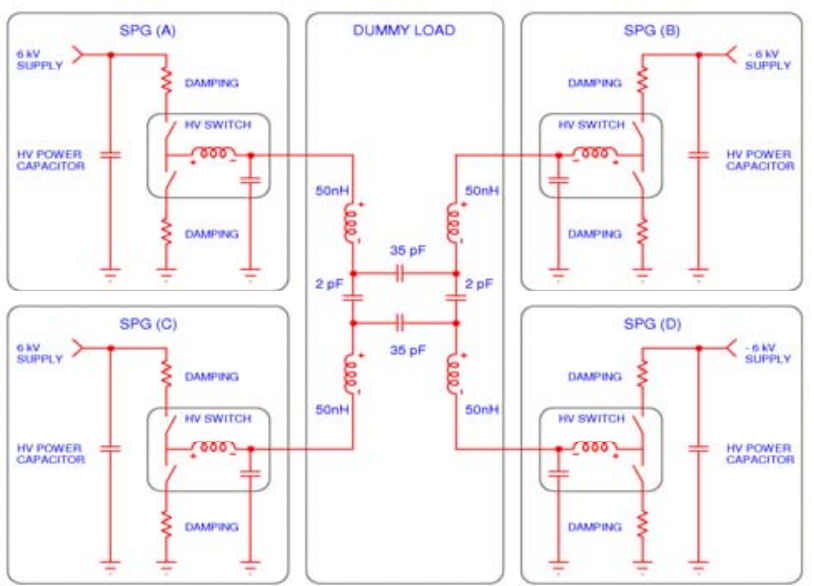

Figure 9: SPG prototype test rig / schematic

The prototype SPG modules will be compact $(100 \mathrm{x}$ $250 \times 280 \mathrm{~mm})$, and fan cooled $(\sim 120 \mathrm{~W})$. The 'lumped element' load will simulate the characteristics of, and be more easily reconfigurable than, a mechanical electrode structure.

Preliminary measurements made on a pre-prototype SPG utilising a commercially available high voltage MOSFET switch module [8], are shown below.

The waveforms shown in Figure 10 indicate that specifications for transition time $(\sim 12 \mathrm{~ns})$ and pulse shape fidelity can be met.

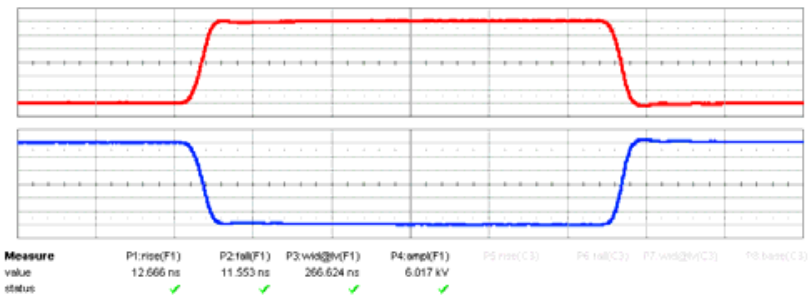

Figure 10: SPG waveforms at $\pm 6.0 \mathrm{kV}$ peak \& $50 \mathrm{~ns} / \mathrm{div}$.

The waveforms shown in Figure 11 demonstrate switch operation at the required PRF of $\sim 1.2 \mathrm{MHz}$. The maximum burst duration at this PRF is limited to $\sim 5 \mu$ s.

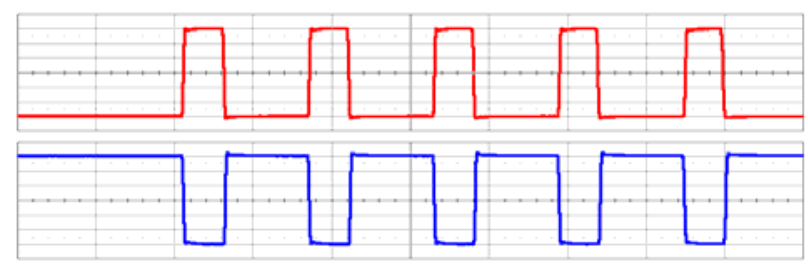

Figure 11: SPG waveforms at $\pm 6.0 \mathrm{kV}$ peak $\& 0.5 \mu \mathrm{s} /$ div.

The waveforms shown in Figure 12 demonstrate switch operation at a PRF of $\sim 100 \mathrm{kHz}$ (PRF limit for this model with $>20$ pulses per burst @ 50Hz).

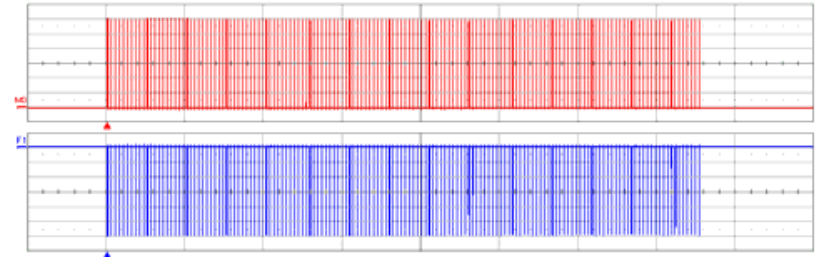

Figure 12: SPG waveforms at $\pm 6.0 \mathrm{kV}$ peak \& $0.2 \mathrm{~ms} /$ div.

The histogram shown in Figure 13 indicates that the requirement for stability in delay ( $\pm 0.5 \mathrm{~ns}$ pk-pk) between the trigger and output pulse can be met.

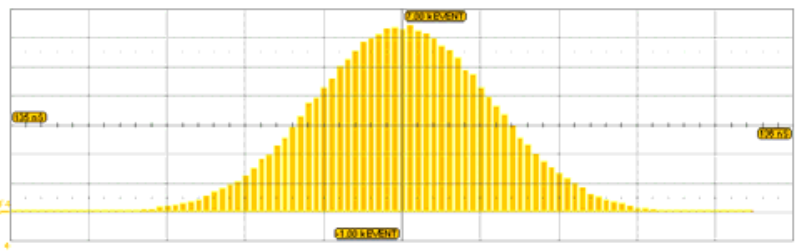

Figure 13: SPG timing jitter at 100ps/div.

\section{SUMMARY}

FPG waveform measurements, following the TLT upgrade, are encouraging. Almost all of the ESS specifications have now been met. A reduction in post pulse aberration and a scheme for the dc restoration of the duty cycle related baseline shift are anticipated.

Waveform measurements on the pre-prototype SPG are also encouraging, as they suggest that an 'off the shelf' high voltage MOSFET switch technology [8] comes close to meeting the requirements of the ESS 'slow' chopper system. A modified version, with a $1.5 \mathrm{~ms}$ burst duration capability at $1.2 \mathrm{MHz}$ PRF is anticipated.

\section{REFERENCES}

[1] M. A. Clarke-Gayther, 'A Fast Beam Chopper for Next Generation High Power Proton Drivers', Proc. of the Ninth EPAC, Lucerne, Switzerland, 5-9 July, 2004, p. 1449-1451.

[2] 'Spallation Neutron Source and other high intensity proton sources', W. Chou, FNAL Report no. FERMILAB-Conf-03/012.

[3] F. Caspers, 'Review of Fast Beam Chopping', Proc. of LINAC 2004, Lubeck, Germany, 16-20 August, 2004, p.294-296.

[4] 'The ESS Project, Volume III Update: Technical Report - Status 2003', ISBN 3-89336-345-9.

[5] M. A. Clarke-Gayther, 'Modulator Systems for the ESS 2.5 MeV Fast Chopper', Proc. of PAC 2001, Chicago, Illinois, USA, p. 4062-4065.

[6] M. A. Clarke-Gayther, 'Slow-wave electrode structures for the ESS $2.5 \mathrm{MeV}$ fast chopper', Proc. of PAC 2003, Portland, Oregon, USA, p. 1473-1475.

[7] Kentech Instruments Ltd., Unit 9, Hall Farm Workshops, South Moreton, Oxfordshire, OX11 9AG, United Kingdom.

[8] Model No. HTS 81-06-GSM-CF-HFS, BEHLKE ELECTRONIC GMBH, Am Auernberg 4, 61476 Kronberg, Germany. 